\title{
Quantitative Analysis of Relationship Between Visual Attention and Eye-Hand Coordination
}

\author{
Attila Kovari ${ }^{1}$, Jozsef Katona ${ }^{1}$, Cristina Costescu ${ }^{2}$ \\ ${ }^{1}$ University of Dunaujvaros, CogInfoCom Based LearnAbility Research Team \\ Tancsics M. 1/A, 2400 Dunaujvaros, Hungary \\ E-mail: \{kovari, katonaj\}@uniduna.hu \\ ${ }^{2}$ Babes-Bolyai University, Faculty of Psychology and Educational Sciences \\ 7 Sindicatelor Street, RO-400029, Cluj-Napoca, Romania \\ E-mail: \{cristina.costescu\}@ubbcluj.ro
}

\begin{abstract}
In perception and activity tasks, continuous visual tracking of the performed activity requires continuous eye motion. Besides writing and reading, the cooperative work of eyes and hands is a key factor when drawing and at certain motions (e.g. ball catching or throwing); during the exact execution of motions, eye-hand coordination has the utmost importance. The development of eye-hand coordination plays a key role in education too, regarding several subjects, i.e. writing, drawing, technique and lifestyle, and of course, at complex motion sequences. Modern info-communication tools play an even more significant role in supporting education, where human-computer interfaces similar to the systems introduced in this paper are very significant. In this paper, by analysing certain features describing computer mouse cursor motion, examined during the execution of the Trail Making Task, what correlation is there between visual attention and eye-hand coordination. Based on the statistical correlation analysis results of data, it was determined, that the fixation parameters of eye and hand motion are in negative correlation with visual attention, while the distance between the look and the mouse cursor's motion are not correlated to each other.
\end{abstract}

Keywords: eye-hand coordination; Trial Making Task; correlation analysis

\section{Introduction}

In perception and activity tasks, continuous visual tracking of the performed activity requires continuous eye motion. Besides writing and reading, the cooperative work of eyes and hands is a key factor when drawing and at certain motions (e.g. ball catching or throwing); during the exact execution of motions, eye-hand coordination has the utmost importance. In education, its development in different tasks has highlighted significance regarding several subjects. Analyses 
related to eye-hand coordination problems play a major role in early detection and proper therapy, specification of development.

More and more studies deal with the analysis of human motion. The human motion's image-based computer observation, recording and evaluation provide several opportunities for example in the development of human-computer interfaces [1] [2], in the exploration of certain motion problems, analysis of motions [3] [4] [5], analysis of certain learning processes [6] or even in humanrobot cooperation, or robot-based rehabilitation [7].

Humans are able to reach and catch target objects, despite different circumstances, even if the object's position changes. This ability is enabled by eye-hand coordination. The simultaneous studies of eye and hand motions are required to understand this behavior. For example, to continuously and precisely track a moving object or line, the appropriate eye-hand coordination is necessary. In such types of tasks, keeping the look close to the target is important; the stable retina is critical for proper control [8].

The internal process performing eye-hand coordination has a complex control, specified by the complex of cognitive abilities [9]. Eye-hand coordination is such a sensory mechanism that controls eye and hand motions as a single unit [10].

In this paper, the eye-hand coordination is analysed during the Trail Making Test. Trail Making Test is widely used to analyse neurocognitive abilities, and to test normal functions [11]. The test primarily serves as a measure of visual searching or scanning, visuospatial sequencing, although, during its execution, rapid eyehand coordination plays a key role. [12].

The analysis of eye-hand movements was examined using the gaze and mouse fixations and average gaze mouse path distance while solving the Trail Making Task. The [13] includes the results of descriptive statistics.

\section{Eye-Hand Coordination}

People have very developed abilities to track and catch moving objects changing their position. This brain-controlled ability is called eye-hand coordination. Eyehand coordination is a complex process because it includes the visual control of both eyes and hands while using eye movements to optimize vision at the same time.

Eye and hand motion analyses are performed mainly regarding fix position targets, and there are fewer studies regarding moving targets [14]. In certain studies, tracking of moving target with the eye was examined [15], whilst in others, the joint tracking of moving target with the eyes and hands [16]. 
Three methods are widely used to analyse human motion: passive, wearable detector and the pointer. In the case of the passive solution, the camera is placed in a fix position, usually opposite to the testing subject; thus, the image area is fix [17]. In the case of a wearable detector, the device is attached to the body, which may be even a wearable camera [18] [19] [20]. The pointer approach is based on that we look to the direction where we would like to do something. The eyetracking method is applicable to monitor the look. In this case, two cameras attached to the head are used, the first camera returns the view seen by its user, while the other monitors the eye motion and determines the look's direction accordingly. Several manufacturers produce such devices to be worn as googles [21].

The eye-hand visual-motoric controlling system implements a closed-loop visual regulation, although such feedforward abilities are required, that help to forecast the motion's track [22]. The internal process performing the eye-hand coordination has a complex control, specified by the complex of cognitive abilities [22]. Eyehand coordination is such a sensory mechanism that controls eye and hand motions as a single unit. The brain has first to solve the geometric transformation between the world perceived by the eyes and the body-centered world to achieve these motions. Second, the brain has to work out a plan how to reach the object and assess the motoric motion of the hand in the coordinate system relative to the body, taking the information and the hand's actual position perceived by the eyes into consideration [23]. Moreover, during the assessment of the motoric motion, the size, shape, motion, and orientation of the object to be caught must be considered.

No united theory exactly describing eye-hand coordination has been worked out yet, and it is not yet clarified either, to what extent does information stored in the memory participate in motion planning. First, according to studies, the brain uses information that is both continuous visual and stored in the memory, depending on their reliability. If continuous visual information is reliable, then the brain primarily relies on it during motion planning [24]. If the reliability of the continuous visual information is getting worse, then the brain starts using information stored in the memory to plan the motion. In the case of worse visibility, the hand motion becomes uncertain.

From the aspect of eye-hand coordination, the direction of the look's fixation is a key factor since prior to catching an object, the look is directed onto the object to be caught for a longer period [25]. Fixations are stable fixed right until catching the object; however, fixations directed to the object are no longer necessary then.

Accordingly, fixations have a triple role in motion planning:

- different fixations are necessary to map the environment and to determine the position of the object to be reached; 
- based on the fixations in the right points, and their sequences, the brain is able to assess the human body's coordinate system relative to the world's coordinate system [25];

- The brain stores the position of the detected objects in the memory and the sequence of positions recorded during fixations, thus enabling motion planning depending on the task to be achieved.

\section{Trial-Making Test}

Trail Making Test (TMT) one of the most widely used tools of neuropsychological examinations. The goal of TMT is to check the visual attention, the processing speed, visual searching, analysis of motoric performance and fast eye-hand coordination. [26] [27] [28].

The PEBL (The Psychology Experiment Building Language) version of the test consists of three parts that must be executed as quick and accurate as possible. During the TMT-A1, 25 randomly assorted numbers must be interconnected in increasing order (1-2-3-4, etc.) (Fig. 1) and similarly with the sequence of the letters (A-B-C-D, etc.) in TMT-A2. TMT-A measures visual attention and scanning, and speed of eye-hand coordination. In the TMT-B, similarly, but the numbers (1-13) and letters $(\mathrm{A}-\mathrm{M})$ alternate sequentially $(1-\mathrm{A}-2-\mathrm{B}-3-\mathrm{C}$, etc.) (Fig. 2). TMT-B additionally, assesses working memory and executive functions. The results of each part are determined by the TMT test solving time and errors. The test solving time expresses the result of visual attention and scanning because the errors in select next cell increase the time of solution.

The Trail-making task's PEBL version is able to run the test according to ,retain original configuration [29], and new tests generated during each new running, although the application of automatically generated tasks is recommended since it enables partial examinations.

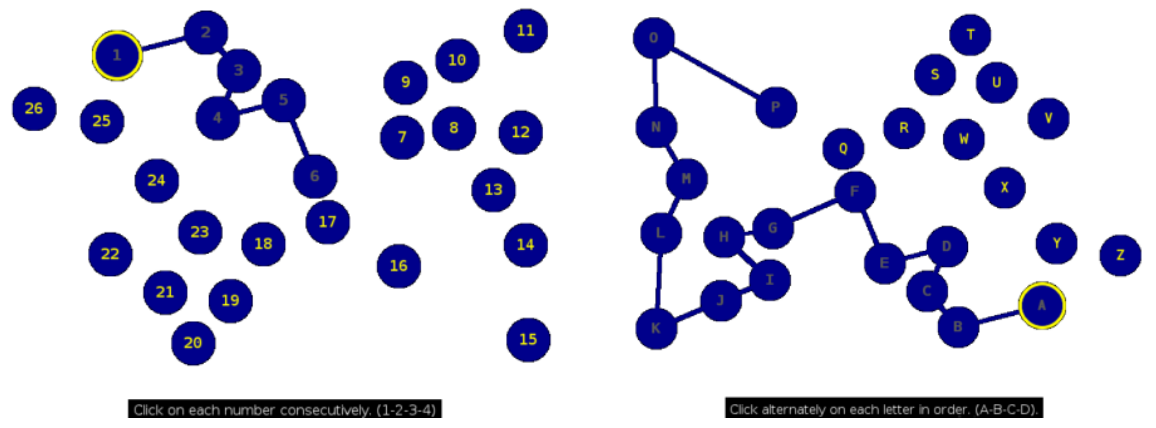

Figure 1

PEBL Trail Making Task Test A1 and A2 


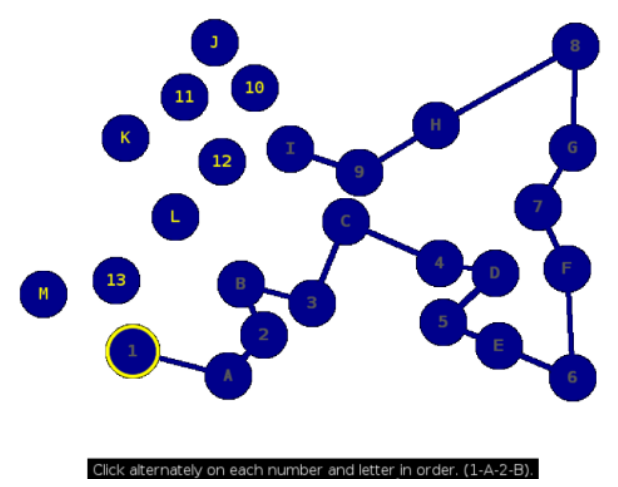

Figure 2

PEBL Trail Making Task Test B

\section{Eye and Mouse Cursor Tracking}

The recording of the eye movement was made using a GP3 Eye Tracker (Fig. 3), which has $0.5-1^{\circ}$ view angle accuracy, $60 \mathrm{~Hz}$ sampling, and is appropriate for general research purposes. The recording and analysis of eye and mouse motion parameters were made using the OGAMA (OpenGazeAndMouseAnalyzer) software. The application records the eye and mouse cursor trail in a database and determines the specific parameters of the motion, and provides other evaluation opportunities too.

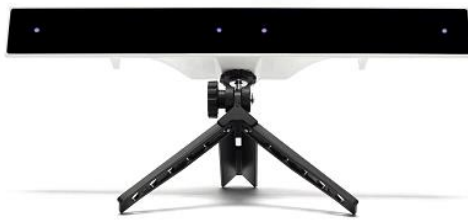

Figure 3

Gazepoint GP3 Eye Tracker 


\section{Methods}

\subsection{Participants}

Eleven men and eight women participated in the TMT - Eye-hand coordination test on a voluntary basis. Their age varied between 10 and 70 years. The age and gender distribution of testing subjects are listed in Table 1.

Table 1

Participants

\begin{tabular}{|c|c|c|}
\hline \multirow{2}{*}{ Age } & \multicolumn{2}{|c|}{$\begin{array}{c}\text { Number of test } \\
\text { subjects }\end{array}$} \\
\cline { 2 - 3 } & Male & Female \\
\hline $10-20$ & 1 & 1 \\
\hline $20-30$ & 3 & 2 \\
\hline $30-40$ & 3 & 2 \\
\hline $40-50$ & 2 & 1 \\
\hline $60-70$ & 2 & 2 \\
\hline Total & $\mathbf{1 1}$ & $\mathbf{8}$ \\
\hline
\end{tabular}

\subsection{Procedures}

During the performance of the PEBL Trail-making task, the eye-tracking system and OGAMA software were applied to record eye-hand coordination data (Fig. 4). During the test, using the Gazepoint Control software establishing communication with the GP3 eye tracker, and the gaze and mouse path was recorded by OGAMA software after the calibration process, thenfurther statistical analysis was performed following the tests. The eye and mouse movement was recorded separately during the three partial tests. The data analysis was carried out offline by statistical methods.

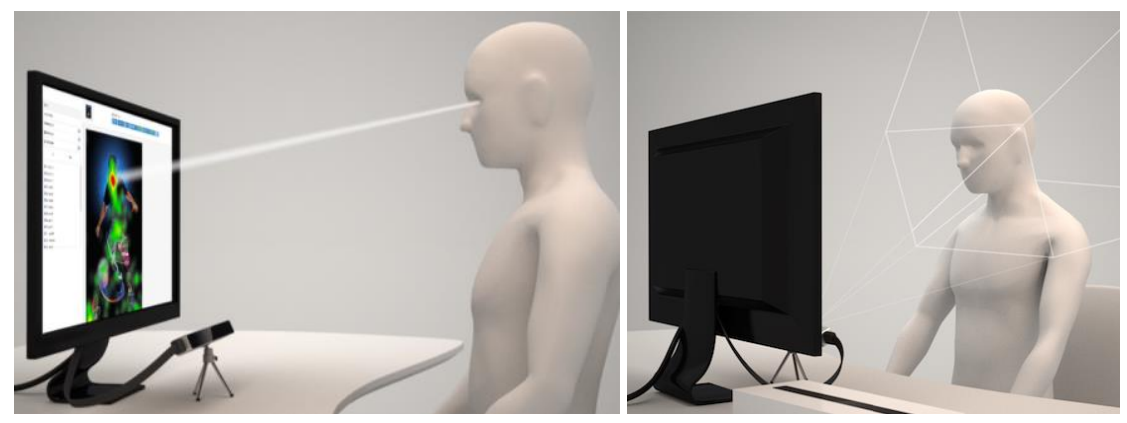

Figure 4

Eye-tracking setup [?] 


\subsection{Analysis}

The variables: gaze fixations, average gaze mouse path distance and mouse fixations are examined because mainly, these parameters are related to eye-mouse motion features in the coordination. These parameters are the dependent and the duration time of the Trail Making Task is the independent variable. These parameters are measured at the ratio level.

The linear relationship is assumed between these examined variables and was examined by scatterplots. Scatterplot was used to plot the dots of examined variables, and then visually inspected the linearity showing the Fit Line in the scatterplots. Outliers are checked using Box plots. Normality is examined of each variable separately by Shapiro-Wilk test and Q-Q plots. In the case of a normally distributed variable, the Pearson correlation is used, if not, the nonparametric Spearman correlation is applied.

\section{Results}

The next chapters summarize the quantitative results of statistical correlation analysis of gaze and mouse tracking parameters while solving TMT-A1, TMT-A2 and TMT-B tests. The results of the three main steps are summarized: investigation of the linear relationship and outliers; normality test; results of Pearson or Spearman correlations.

However, it should be mentioned that OGAMA software also provides the opportunity to conduct qualitative tests, for example, attention map or scan path of gaze or mouse cursor motion (Fig. 5).

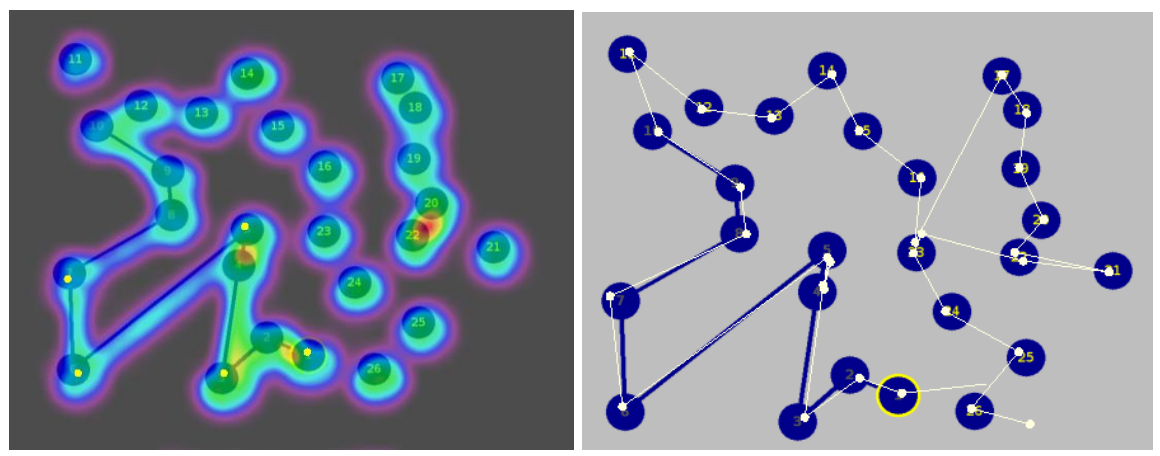

Figure 5

Example gaze attention map and scan paths of mouse cursor while solving TMT-A1 [?] 


\subsection{Correlation Analysis for TMT-A1 Task}

\subsubsection{Investigation the Linear Relationship and Outliers of TMT-A1 Task}

Fig. 6 shows the scatter plots of gaze fixations count, average gaze mouse path distance and mouse fixations count. As shown in the plots, the gaze and mouse fixations counts are in a linear relationship with TMT-A1 Task solving time, but average gaze mouse path distance dots do not fit a line. According to Box plots (Fig. 7), the results of Test subject 13, 18 and 19 consists outliers (showing "*”) which should, therefore, be ignored in further analysis.
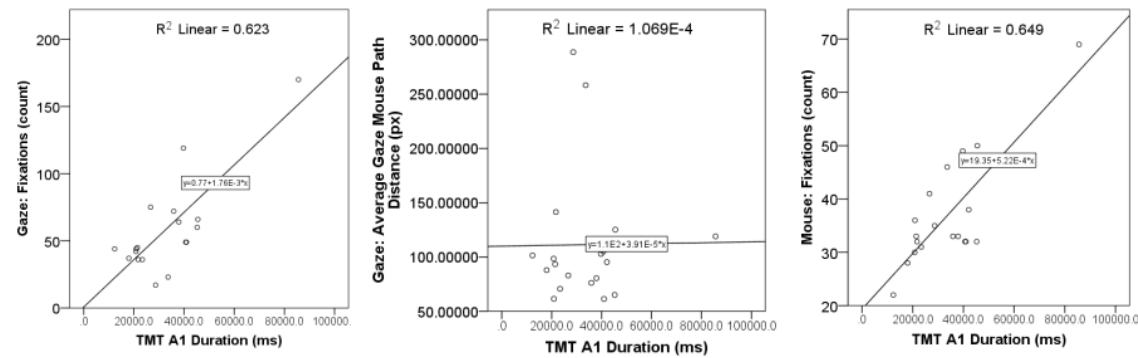

Figure 6

Scatter plot results of TMT-A1 gaze-mouse parameters
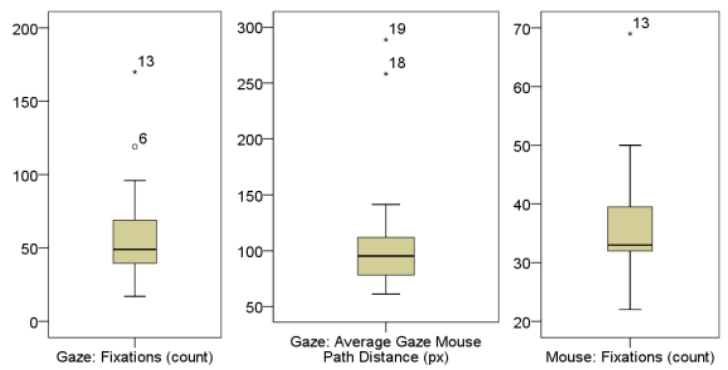

Figure 7

Box plot results of TMT-A1 gaze-mouse parameters

\subsubsection{Normality Test of TMT-A1 Task}

Fig. 8 shows the Q-Q plots of the examined gaze fixations count, average gaze mouse path distance and mouse fixations count parameters. The values do not fit well on the line $y=x$, so the normal distribution is not approximated for gaze and mouse fixations; however, it can be approximated for average gaze mouse path distance. The quantitative results in Table 2 confirm this statement because the Sig. of Shapiro-Wilk test is less than 0.05 for gaze and mouse fixations. 

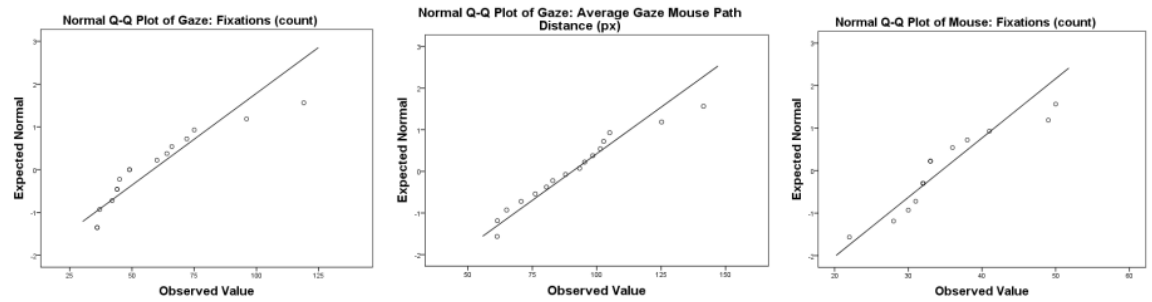

Figure 8

Q-Q plots of TMT-A1 gaze-mouse parameters

Table 2

Tests of Normality for TMT-A1 gaze-mouse parameters

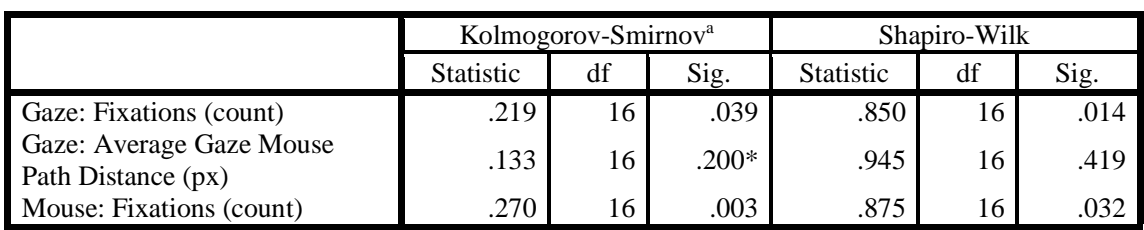

*. This is a lower bound of the true significance.

a. Lilliefors Significance Correction

\subsubsection{Correlations of TMT-A1 Task}

Based on the results of the normality test Pearson correlation is calculated for Average Gaze Mouse Path Distance, and Spearman correlation for gaze and mouse fixations while the independent variable is TMT solving duration time (Table 3).

Table 3

Correlations of TMT-A1 gaze-mouse parameters

\begin{tabular}{|ll|r|r|r|}
\hline & $\begin{array}{c}\text { Gaze: } \\
\text { Fixations } \\
\text { (count) }\end{array}$ & $\begin{array}{c}\text { Gaze: Average } \\
\text { Gaze Mouse Path } \\
\text { Distance (px) }\end{array}$ & $\begin{array}{c}\text { Mouse: } \\
\text { Fixations } \\
\text { (count) }\end{array}$ \\
\hline $\begin{array}{ll}\text { TMT Duration } \\
(\mathrm{ms})\end{array}$ & $\begin{array}{l}\text { Correlation } \\
\text { Coefficient }\end{array}$ & $.571^{*}$ & .194 & $.508^{*}$ \\
& Sig. (2-tailed) & .013 & .456 & .031 \\
& $\mathrm{~N}$ & 18 & 17 & 18 \\
\hline
\end{tabular}

*. Correlation is significant at the 0.05 level (2-tailed)

The value of correlations showing medium correlation and significant relationship for Gaze $(\mathrm{r}=.571, \mathrm{n}=18, \mathrm{p}=.005)$ and Mouse Fixations $(\mathrm{r}=.508, \mathrm{n}=18, \mathrm{p}=$ .005) which is statistically significant and smallweak relationship for Average Gaze Mouse Path Distance $(\mathrm{r}=.194, \mathrm{n}=17, \mathrm{p}=.456)$. 


\subsection{Correlation Analysis for TMT-A2 Task}

\subsubsection{Investigation the Linear Relationship and Outliers of TMT-A2 Task}

Fig. 9 shows the scatter plots of gaze fixations count, average gaze mouse path distance and mouse fixations count. As shown in the plots, the gaze and mouse fixations counts are in a linear relationship with TMT-A2 Task solving time, but average gaze mouse path distance dots do not fit a line. According to Box plots the results (Fig. 10) of Test subject 18 and 19 consists outliers (showing "*") which should, therefore, be ignored in further analysis.
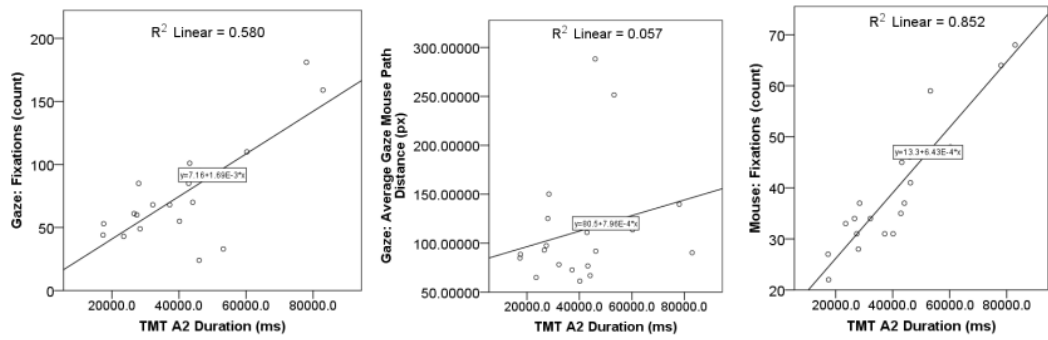

Figure 9

Scatter plot results of TMT-A2 gaze-mouse parameters
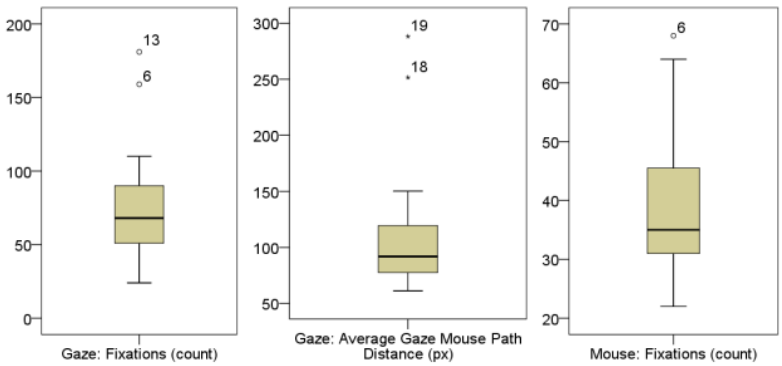

Figure 10

Box plot results of TMT-A2 gaze-mouse parameters

\subsubsection{Normality Test of TMT-A2 Task}

Fig. 11 shows the Q-Q plots and normal distribution is not well approximated for gaze and mouse fixations; however, it can be approximated for average gaze mouse path distance. The quantitative results in Table 4 confirm this statement because the Sig. of Shapiro-Wilk test is less than 0.05 for gaze and mouse fixations. 

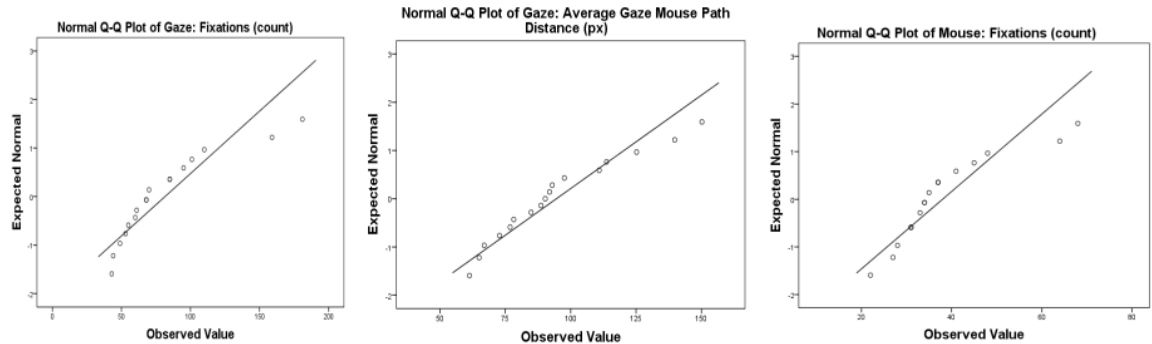

Figure 11

Q-Q plots of TMT-A2 gaze-mouse parameters

Table 4

Tests of Normality for TMT-A2 gaze-mouse parameters

\begin{tabular}{|c|c|c|c|c|c|c|}
\hline & \multicolumn{3}{|c|}{ Kolmogorov-Smirnov $^{\mathrm{a}}$} & \multicolumn{3}{|c|}{ Shapiro-Wilk } \\
\hline & Statistic & df & Sig. & Statistic & df & Sig. \\
\hline Gaze: Fixations (count) & .206 & 17 & .055 & .834 & 17 & .006 \\
\hline $\begin{array}{l}\text { Gaze: Average Gaze Mouse Path } \\
\text { Distance (px) }\end{array}$ & .171 & 17 & .198 & .929 & 17 & .211 \\
\hline Mouse: Fixations (count) & .238 & 17 & .011 & .848 & 17 & .010 \\
\hline
\end{tabular}

a. Lilliefors Significance Correction

\subsubsection{Correlations of TMT-A2 Task}

Based on the results of the normality test Pearson correlation is calculated for Average Gaze Mouse Path Distance, and Spearman correlation for gaze and mouse fixation while the independent variable is TMT solving duration time (Table 5).

Table 5

Correlations of TMT-A2 gaze-mouse parameters

\begin{tabular}{|ll|r|r|r|}
\hline & $\begin{array}{c}\text { Gaze: } \\
\text { Fixations } \\
\text { (count) }\end{array}$ & $\begin{array}{c}\text { Gaze: Average } \\
\text { Gaze Mouse Path } \\
\text { Distance (px) }\end{array}$ & $\begin{array}{c}\text { Mouse: } \\
\text { Fixations } \\
\text { (count) }\end{array}$ \\
\hline $\begin{array}{l}\text { TMT Duration } \\
(\mathrm{ms})\end{array}$ & Correlation & $.537^{*}$ & .227 & $.892^{* *}$ \\
& Coefficient & .018 & .382 & .000 \\
& Sig. (2-tailed) & 19 & 17 & 19 \\
\hline
\end{tabular}

*. Correlation is significant at the 0.05 level (2-tailed).

**. Correlation is significant at the 0.01 level (2-tailed)

The value of correlations showing medium correlation and significant relationship for Gaze $(\mathrm{r}=.537, \mathrm{n}=19, \mathrm{p}=.018)$ and high correlation, strong relationship for Mouse Fixations $(r=.892, n=19, p=.000)$ which is statistically significant and small relationship for Average Gaze Mouse Path Distance $(r=.227, n=17, p=$ $.382)$. 


\subsection{Correlation Analysis for TMT-B Task}

\subsubsection{Investigation the Linear Relationship and Outliers of TMT-B Task}

Fig. 12 shows the scatter plots of gaze fixations count, average gaze mouse path distance and mouse fixations count. As shown in the plots, the gaze and mouse fixations counts are in a linear relationship with B Task solving time, but average gaze mouse path distance dots do not fit a line. According to Box plots, the results (Fig. 13) of Test subject 19 consists of outliers (showing "*") which should, therefore, be ignored in further analysis.
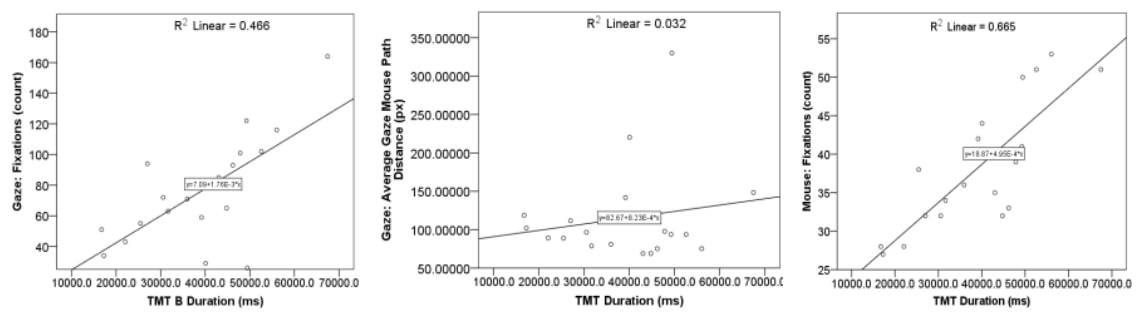

Figure 12

Scatter plot results of Trail Making Task B variables
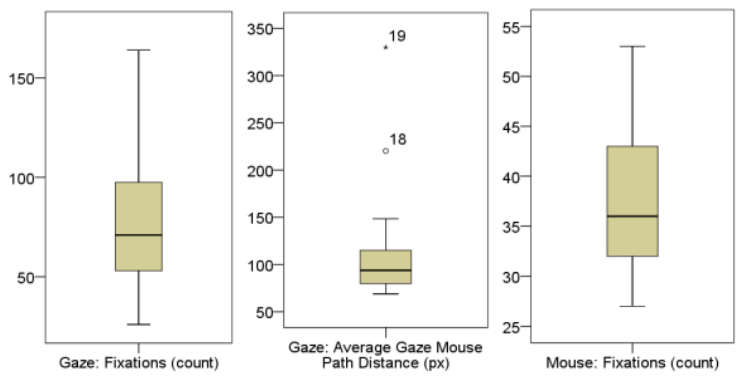

Figure 13

Box plot results of Trail Making Task B variables

\subsubsection{Normality Test of TMT-B Task}

Fig. 14 shows the Q-Q plots and normal distribution is approximated for gaze and mouse fixations; however, it can not be approximated for average gaze mouse path distance. The quantitative results in Table 6 confirm this statement because the Sig. of Shapiro-Wilk test is less than 0.05 for the average gaze mouse path distance. 

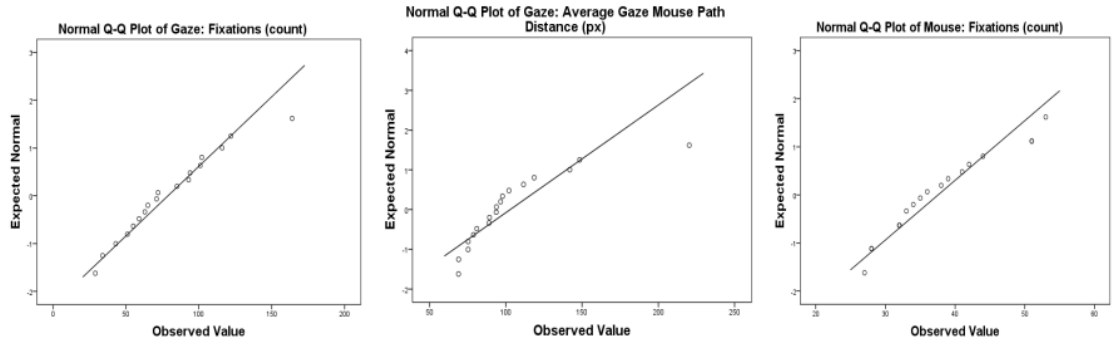

Figure 14

Q-Q plots of Trail Making Task A2 variables

Table 6

Tests of Normality for TMT-A2 gaze-mouse parameters

\begin{tabular}{|l|r|r|r|r|r|r|}
\hline & \multicolumn{3}{|c|}{ Kolmogorov-Smirnov $^{\mathrm{a}}$} & \multicolumn{3}{c|}{ Shapiro-Wilk } \\
\cline { 2 - 7 } & Statistic & \multicolumn{1}{c|}{$\mathrm{df}$} & \multicolumn{1}{c|}{ Sig. } & Statistic & \multicolumn{1}{c|}{$\mathrm{df}$} & \multicolumn{1}{c|}{ Sig. } \\
\hline Gaze: Fixations (count) & .134 & 18 & $.200^{*}$ & .957 & 18 & .548 \\
Gaze: Average Gaze Mouse Path & .231 & 18 & .012 & .778 & 18 & .001 \\
Distance (px) & .132 & 18 & $.200^{*}$ & .919 & 18 & .126 \\
Mouse: Fixations (count) & & &
\end{tabular}

*. This is a lower bound of the true significance.

a. Lilliefors Significance Correction

\subsubsection{Correlations of TMT-B Task}

Based on the results of the normality test Spearman correlation is calculated for Average Gaze Mouse Path Distance, and Pearson correlation for gaze and mouse fixation while the independent variable is TMT solving duration time (Table 7).

Table 7

Correlations of TMT-B gaze-mouse parameters

\begin{tabular}{|ll|r|r|r|}
\hline & \multicolumn{1}{|c|}{$\begin{array}{c}\text { Gaze: } \\
\text { Fixations } \\
\text { (count) }\end{array}$} & $\begin{array}{c}\text { Gaze: Average } \\
\text { Gaze Mouse Path } \\
\text { Distance (px) }\end{array}$ & $\begin{array}{c}\text { Mouse: } \\
\text { Fixations } \\
\text { (count) }\end{array}$ \\
\hline $\begin{array}{l}\text { TMT Duration } \\
(\mathrm{ms})\end{array}$ & $\begin{array}{l}\text { Correlation } \\
\text { Coefficient }\end{array}$ & $.683^{* *}$ & .164 & $.815^{* *}$ \\
& Sig. (2-tailed) & .001 & .515 & .000 \\
& $\mathrm{~N}$ & 19 & 18 & 19 \\
\hline
\end{tabular}

**. Correlation is significant at the 0.01 level (2-tailed).

The value of correlations showing medium correlation and significant relationship for Gaze $(\mathrm{r}=.683, \mathrm{n}=19, \mathrm{p}=.001)$ and high correlation, strong relationship for Mouse Fixations $(\mathrm{r}=.815, \mathrm{n}=19, \mathrm{p}=.000)$ which is statistically significant and small relationship for Average Gaze Mouse Path Distance $(r=.164, n=18, p=$ $.515)$. 


\section{Discussion}

The rapid execution of the Trail Making Task that affected the test time and served as the independent variable of the test mainly depends on the visual attention and rapid and quick eye-hand coordination. Since the quick solution of the task depends on the visual searching, the precise and rapid motion of the look from the fixation directed onto the next target to the mouse cursor target, the test was aimed on the evaluation of this activity. In the execution of the appropriate task, the coordination of eyes and hand depends on the visual attention, search, primarily the number of the look's fixation and moving the mouse cursor into position quickly and precisely, the test primarily focused on them, to analyse the fixations as targets, and on the average distance regarding moving of the look relative to the mouse cursor. By the post-processing of eye motion and mouse cursor motion recorded during the execution of the three versions of the Trail Making Task, the aforementioned parameters were received. Based on the results, the eye movements and the mouse cursor motion's fixation number is in medium or strong relevance with the test's execution time, which was proven to be significant. Since there is an opposite relationship between the test's execution time and the level of visual attention, the fixation numbers negatively correlate with visual attention. These results refer to that by more efficient visual attention, quicker visual searching, the shorter task execution reduces the number of fixations of both eye motion and mouse cursor movement, so the look is quickly directed to the next target without recording intermediate points. In case of the distance between the look and the mouse cursor, however, a negligible correlation was experienced, meaning that the trail of the look and the mouse cursor compared to each other does not significantly depend on the visual attention level and the searching agility. Relative to the look's faster or slower motion; the mouse cursor moves in similar speed, so closer visual attention, quicker visual searching, the eye's more purposeful motion involves the quicker and more precise motion of the mouse cursor, i.e. the hand. Based on this, the agility of the eye-hand coordination is in a positive relationship with visual attention, so these factors correlate with each other. For the future, it is also appropriate to examine how age influences the results.

\section{Conclusions}

In this paper the visual attention and hand coordination was examined by analyzing certain features of eye- and computer mouse cursor motion. Based on the statistical correlation analysis results of data, it was determined, that the fixation parameters of eye and hand motion are in negative correlation with visual attention, while the distance between the look and the mouse cursor's motion are not correlated to each other. Visual attention and proper eye-hand coordination have key significance from the aspect of the proper execution of several human activities like tool usage, manual machining for example; thus the recognition of potentially occurring coordination problems, its development, reduction of 
problems are all important factors. The results may help to detect eye-hand coordination problems, which may be the cause of problems occurring in writing and drawing abilities, this helping to specify the necessity of eye-hand coordination development. According to the above, the development of eye-hand coordination plays a key role in education too, regarding several subjects, i.e. writing, drawing, technique and lifestyle, and of course, at complex motion sequences. Modern infocommunication tools play an even more significant role in supporting education [30]-[35], where eye-hand coordination is important factor such as gamification [36], VR based education [37]-[43] project-based teamwork [44] or other visual, interactive systems [45]-[49].

\section{Acknowledgement}

This research was supported by EFOP-3.6.1-16-2016-00003 grants, Establishment long-term R \& D \& I process at the University of Dunaujvaros.

\section{References}

[1] K. Wei and Y.-P. Guan: Eye-hand Coordination based Human-Computer Interaction, International Journal of Signal Processing, Image Processing and Pattern Recognition, Vol. 9, No. 10, 2016, pp. 205-216

[2] A. Murata and K. Inoue: A Method to Measure Eye-Hand Coordination for Extracting Skilled Elements-Simultaneous Measurement of Eye-Gaze and Hand Location, Computer Technology and Application, Vol. 5, No. 2, 2014, pp. 73-82

[3] L. Sigal and M. J. Black: Guest Editorial: State of the Art in Image- and Video-Based Human Pose and Motion Estimation, International Journal of Computer Vision, Vol. 87, No. 1-2, 2009, pp. 1-3

[4] R. Poppe: A survey on vision-based human action recognition, Image and Vision Computing, Vol. 28, No. 6, 2010, pp. 976-990

[5] H. Zhou and H. Hu: Human motion tracking for rehabilitation-A survey, Biomedical Signal Processing and Control, Vol. 3, No. 1, 2008, pp. 1-18

[6] T. Ujbányi: Examination of eye-hand coordination using the computer mouse and hand tracking cursor control," $20189^{\text {th }}$ IEEE International Conference on Cognitive Infocommunications (CogInfoCom), Budapest, Hungary, 2018, pp. 353-354

[7] K. Sakita, K. Ogawa, S. Murakami, K. Kawamura, and K. Ikeuchi: Flexible cooperation between human and robot by interpreting human intention from gaze information, 2004 IEEE/RSJ International Conference on Intelligent Robots and Systems (IROS), 2004, pp. 846-851

[8] Danion, Frederic R., Flanagan, J. Randall: Different gaze strategies during eye versus hand tracking of a moving target, Scientific reports, Vol. 8, No. $1,2018,10059$ 
[9] J. Crawford, W. Medendorp and J. Marotta: Spatial Transformations for Eye-Hand Coordination, Journal of Neurophysiology, Vol. 92, 2004, No. 1, pp. 10-19

[10] C. A. Bueno, M. R. Jarvis, A. P. Batista, and R. A. Andersen: Direct visuomotor transformations for reaching, Nature, Vol. 416, No. 6881, 2002, pp. 632-636

[11] Salthouse Timothy A.: What cognitive abilities are involved in trail-making performance?, Intelligence, Vol. 39, No. 4, 2011, pp. 222-232

[12] Sara Cavaco et al.: Trail Making Test: Regression-based Norms for the Portuguese Population, Archives of Clinical Neuropsychology, Oxford University Press, Vol. 28, No. 2, 2013, pp. 189-198

[13] Attila Kovari et al.: Examination of Gaze Fixations Recorded during the Trail Making Test, $201910^{\text {th }}$ IEEE International Conference on Cognitive Infocommunications (CogInfoCom), 2019, in press

[14] Li, Yuhui, Yong Wang, and He Cui.: Eye-hand coordination during the flexible manual interception of an abruptly appearing, moving target, Journal of neurophysiology, Vol. 119, No. 1, 2017, pp. 221-234

[15] Barnes, Graham R.: Cognitive processes involved in smooth pursuit eye movements." Brain and cognition, 68, No. 3, 2008, pp. 309-326

[16] Niehorster, Diederick C., Wilfred WF Siu, and Li Li.: Manual tracking enhances smooth pursuit eye movements, Journal of Vision, Vol. 15, No. 15,2015 , p. 11

[17] P. Turaga, R. Chellappa, V. S. Subrahmanian, and O. Udrea: Machine Recognition of Human Activities: A Survey, IEEE Transactions on Circuits and Systems for Video Technology, Vol. 18, No. 11, 2008, pp. 1473-1488

[18] S. Mann: WearCam' (The wearable camera): personal imaging systems for long-term use in wearable tetherless computer-mediated reality and personal photo/video graphics memory prosthesis, Digest of Papers. Second International Symposium on Wearable Computers, 1998, pp. 124-131

[19] J. Healey and R. W. Picard: StartleCam: a cybernetic wearable camera, Digest of Papers. Second International Symposium on Wearable Computers, 1998, pp. 42-49

[20] H. Yamazoe: Head and shoulders pose estimation using a body-mounted camera, $201714^{\text {th }}$ International Conference on Ubiquitous Robots and Ambient Intelligence (URAI), 2017, pp. 142-145

[21] Tobii AB: Tobii Pro Glasses 2, 2019 [Online] Available: https://www.tobiipro.com/product-listing/tobii-pro-glasses-2/ [Accessed: Jun. 1, 2019] 
[22] J. Crawford, W. Medendorp and J. Marotta: Spatial Transformations for Eye-Hand Coordination, Journal of Neurophysiology, Vol. 92, No. 1, 2004, pp. 10-19

[23] C. A. Bueno, M. R. Jarvis, A. P. Batista, and R. A. Andersen: Direct visuomotor transformations for reaching, Nature, Vol. 416, No. 6881, 2002, pp. $632-636$

[24] A.-M. Brouwer and D. C. Knill: The role of memory in visually guided reaching," Journal of Vision, Vol. 7, 2007, No. 5, p. 6

[25] Crawford, J. Douglas; Medendorp, W. Pieter; Marotta, Jonathan J.: Spatial transformations for the eye-hand coordination, Journal of neurophysiology, Vol. 92, No. 1, 2004, pp. 10-19

[26] K. Arbuthnott and J. Frank: Trail Making Test, Part B as a Measure of Executive Control: Validation Using a Set-Switching Paradigm, Journal of Clinical and Experimental Neuropsychology, Vol. 22, No. 4, 2000, pp. 518528

[27] A. Szöke, F. Schürhoff, F. Mathieu, A. Mary, S. Ionescu, and M. Leboyer: Tests of executive functions in first-degree relatives of schizophrenic patients: a meta-analysis, Psychological Medicine, Vol. 35, No. 6, 2004, pp. $771-782$

[28] Sperandeo, R., Maldonato, M., Baldo, G., \& Dell'Orco, S.: Executive functions, temperament and character traits: A quantitative analysis of the relationship between personality and prefrontal functions, $20167^{\text {th }}$ IEEE International Conference on Cognitive Infocommunications (CogInfoCom), 2016, pp. 000043-000048

[29] R. M. Reitan: Validity of the Trail Making Test as an Indicator of Organic Brain Damage, Perceptual and Motor Skills, Vol. 8, No. 3, 1958, pp. 271276

[30] Elod Gogh, Attila Kovari: Examining the relationship between lifelong learning and language learning in a vocational training institution, Journal of Applied Technical and Educational Sciences, Vol. 8, No. 1, 2018, pp. 52-69

[31] Naif M. Algashaam: Teamwork vs. Individual Responsibility, International Journal of Scientific and Engineering Research, Vol. 6, No. 10, pp. 286288,2015

[32] Elod Gogh, Attila Kovari: Metacognition and Lifelong Learning: A survey of secondary school students, $9^{\text {th }}$ IEEE International Conference on Cognitive Infocommunications, 2018, pp. 271-276

[33] Tibor, U. et al.: ICT Based Interactive and Smart Technologies in Education - Teaching Difficulties. International Journal of Management and Applied Science, Vol. 3, No. 10, 2017, pp. 72-77 
[34] Krisztina Erdélyi: How Information Technology Helps to Mitigate Difficulties Occurred In: Teaching Intercultural Groups, ICETA $201210^{\text {th }}$ IEEE International Conference on Emerging eLearning Technologies and Applications, Stará Lesná, The High Tatras, Slovakia, November 8-9, 2012, p. 97

[35] E. Gogh, A. Kovari, Experiences of Self-regulated Learning in a Vocational Secondary School, Journal of Applied Technical and Educational Sciences, Vol. 9, No. 2, 2019, pp. 72-86

[36] Cecilia Sik-Lanyi, Veronika Szucs, Shervin Shirmohammadi, Petya Grudeva, Boris Abersek, Tibor Guzsvinecz, Karel Van Isacker: How to Develop Serious Games for Social and Cognitive Competence of Children with Learning Difficulties, Acta Polytechnika Hungarica, Vol. 16, No. 6, 2019, pp. 149-169

[37] T. Guzsvinecz, Cs. Kovacs, Dominich Reich, V. Szucs, C. Sik-Lanyi: Developing a virtual reality application for the improvement of depth perception, $9^{\text {th }}$ IEEE International Conference on Cognitive Infocommunications, 2018, pp. 17-22

[38] Cecilia Sik-Lányi,Veronika Szücs, Janet Stark: Virtual reality environments development for aphasic clients, International Journal of Stroke, 2014, pp 241-250

[39] Attila Kovari: CogInfoCom Supported Education: A review of CogInfoCom based conference papers, Proceedings of the $9^{\text {th }}$ IEEE International Conference on Cognitive Infocommunications (CogInfoCom), 2018, pp. 233-236

[40] Ildikó Horváth: Evolution of teaching roles and tasks in VR/AR-based education, Proceedings of the $9^{\text {th }}$ IEEE International Conference on Cognitive Infocommunications (CogInfoCom), Budapest, 2018, pp. 355360

[41] Laszlo Bognar, Eva Fancsikne, Peter Horvath, Antal Joos, Balint Nagy, Gyorgyi Strauber: Improved learning environment for calculus courses, Journal of Applied Technical and Educational Sciences, Vol. 8, No. 4, 2018, pp. 35-43

[42] Adam Csapo, Ildiko Horvath, Peter Galambos and Peter Baranyi: VR as a Medium of Communication: from Memory Palaces to Comprehensive Memory Management, Proceedings of the $9^{\text {th }}$ IEEE International Conference on Cognitive Infocommunications (CogInfoCom), Budapest, 2018, pp. 389-394

[43] Ildiko Horvath: Innovative engineering education in the cooperative VR environment, Proceedings of the $7^{\text {th }}$ IEEE Conference on Cognitive Infocommunications (CogInfoCom), Wrocław, 2016, pp. 359-364 
[44] Robert Pinter, Sanja Maravic Cisar: Measuring Team Member Performance in Project Based Learning, Journal of Applied Technical and Educational Sciences, Vol. 8, No. 4, 2018, pp. 22-34

[45] Ilona Heldal, Carsten Helgesen: The Digital HealthLab: Supporting Interdisciplinary Projects in Engineering and in Health Education, Journal of Applied Technical and Educational Sciences, Vol. 8, No. 4, 2018, pp. 421

[46] Gergely Sziladi, et al.: The analysis of hand gesture based cursor position control during solve an IT related task, $8^{\text {th }}$ IEEE International Conference on Cognitive Infocommunications, 2017, pp. 413-418

[47] Tibor Guzsvinecz, Veronika Szucs, Cecilia Sik-Lanyi: Suitability of the Kinect Sensor and Leap Motion Controller - A Literature Review, Sensors, Vol. 19, No. 5, 2019, pp. 1072-1097

[48] Veronika Szucs, Tibor Guzsvinecz, Attila Magyar: Improved algorithms for movement pattern recognition and classification in physical rehabilitation, $10^{\text {th }}$ IEEE International Conference on Cognitive Infocommunications, 2019, pp. 417-424

[49] Veronika Szucs, Gyorgy Karolyi, Amdras Tatar, Attila Magyar: Voice Controlled Humanoid Robot based Movement Rehabilitation Framework, $9^{\text {th }}$ IEEE International Conference on Cognitive Infocommunications, 2018, pp. 191-196 